Schutijser, B.C.F.M., Jongerden, I.P., Klopotowska, J.E., Portegijs, S., Bruijne, M.C. de, Wagner, C.Double checking injectable medication administration: Does the protocol fit clinical practice? Safety Science: 118 (2019) p. 853-860

\author{
Postprint version $\quad: \quad 1.0$ \\ Journal website $\quad:$ https://www.sciencedirect.com/science/article/pii/S0925753519302000 \\ Pubmed link \\ DOI \\ : $\quad$ https://doi.org/10.1016/j.ssci.2019.06.026
}

This is a Nivel certified Post Print, more info at nivel.nl

\title{
Double checking injectable medication administration: Does the protocol fit clinical practice?
}

\author{
Bernadette Clara Francisca Maria Schutijser ${ }^{\mathrm{a}, *}$, Irene Paulien Jongerden ${ }^{\mathrm{a}}$, \\ Joanna Ewa Klopotowska ${ }^{b}$, Suzanne Portegijs ${ }^{c}$, Margaretha Christine de \\ Bruijne $^{\mathrm{a}}$, Cordula Wagner ${ }^{\mathrm{a}, \mathrm{c}}$
}

a Amsterdam UMC, Vrije Universiteit Amsterdam, Department of Public and Occupational Health, Amsterdam Public Health Research Institute, De Boelelaan 1117, Amsterdam, Netherlands

b Amsterdam UMC, University of Amsterdam, Department of Medical Informatics, Amsterdam Public Health Research Institute, Meibergdreef 9, Amsterdam, Netherlands

c Netherlands Institute for Health Services Research (NIVEL), PO Box 1568, 3500 BN Utrecht, Netherlands

\begin{abstract}
Objectives: It is unclear how nurses adjust the double check during injectable medication administration andguarantee patient safety. We used the Functional Resonance Analysis Method (FRAM) to determine the fit betweenthe double check according to the protocol (work-as-imagined) and clinical practice (work-as-done). Weaimed to learn about process variation in order to optimize safety during injectable medication administration.

Methods: A qualitative study (February-July 2018) with semi-structured group interviews. An internal medicineand a surgery ward of two Dutch hospitals participated (four wards total). We interviewed nurses about injectable medication administration practices, based on prior observations. A work-as-done model was constructedfor each hospital. The work-as-imagined model was based on the Dutch protocol for safe injectablemedication administration.

Results: A total of 27 nurses were interviewed. In both hospitals, nurses split the double check into a digital andphysical check to improve workflow. The digital check was routinely conducted. For the physical check, nursesmade their own risk-impact analysis and assessed staffing, familiarity with the medication, severity of sideeffects, type of
\end{abstract}

\footnotetext{
* Corresponding author at: Amsterdam UMC, Vrije Universiteit Amsterdam, Department of Public and Occupational Health, Amsterdam Public Health Research Institute, Van der Boechorststraat 7, 1081 BT Amsterdam, Netherlands.

E-mail address: b.schutijser@vumc.nl (B.C.F.M. Schutijser).
} 
Schutijser, B.C.F.M., Jongerden, I.P., Klopotowska, J.E., Portegijs, S., Bruijne, M.C. de, Wagner, C.Double checking injectable medication administration: Does the protocol fit clinical practice? Safety Science: 118 (2019) p. 853-860

medication and administration route and the patients' medical condition. Based upon thesecriteria, nurses decided to conduct the physical double check or not.

Conclusions: We identified a lack of fit between work-as-imagined and work-as-done. Nurses adjust the double check in practice by assessing the patients' and wards' situation. It is unknown whether this variability also causes patient harm. We recommend to reconsider to what extent practice variation is acceptable and safe.

\section{Introduction}

Over $90 \%$ of all hospitalized patients receive some form of injectable medication, such as intravenous infusion, subcutaneous or intramuscular injections (Institute for Safe Medication Practices (ISMP), 2015). These medications are of great value in the treatment of diseases because of their immediate therapeutic effect. However, administering them has also a high risk for patient harm, since medication errors with injectable medication are often irreversible. The likelihood of at least one error, such as wrong dose or wrong time, during the whole injectable medication process is 73\% (McDowell et al., 2010). Therefore, based on the 'five rights' of safe medication administration (right patient, drug, dose, route, and time) (Hughes and Blegen, 2008), protocols for safe injectable medication administration have been established internationally. In The Netherlands, such a protocol has been implemented in 2009 and includes 25 proceedings (VMS, 2009).

Yet, compliance with the protocol proceedings for safe injectable medication administration is low (Alemanni et al., 2010; Barthelemy et al., 2013; Choo et al., 2013; Schilp et al., 2014; Schutijser et al., 2018; Westbrook et al., 2011). Especially the compliance with the double check proceeding is between 45 and 90\% and needs improvement (Alsulami et al., 2014; Kellett and Gottwald, 2015; Schilp et al., 2014; Schutijser et al., 2018). The double check is defined as "a procedure that requires two qualified health care professionals, usually nurses, who independently check the medication before administration to patients" (Alsulami et al., 2012). Nurses see the double check as a way to prevent medication errors (Schwappach et al., 2018). Compliance with the double check proceeding in The Netherlands did not change over the years (52\% in 2012 and 47\% in 2016) (Schutijser et al., 2018). Especially logistical factors such as staff shortage lead to low compliance (Alsulami et al., 2012; Schutijser et al., 2018).

While previous studies focused only on compliance and the absence of the double check (Alemanni et al., 2010; Alsulami et al., 2014; Schwappach et al., 2016; Sheu et al., 2009), extra understanding is needed about how nurses actually conduct the double check proceeding in everyday clinical practice. After all, most of the work seems to be carried out safely and without harming patients. A deeper understanding may reveal workarounds or adaptation of the proceeding while still guaranteeing patient safety. Furthermore, it may reveal a lack of fit between the protocol (work-as-imagined) and daily clinical practice (work-as-done) and provide possibilities for organizational learning to reduce the gap between work-as-imagined and work-asdone (Furniss et al., 2018).

Previous studies on other proceedings have used The Functional Resonance Analysis Method (FRAM) to evaluate complex clinical processes (Damen et al., 2018; Laugaland et al., 2014; Raben et al., 2018). Protocols are often implemented with the Safety-I idea of preventing errors, whereby compliance is the outcome measure (Clay-Williams et al., 2015). The lack of fit can arise because of insufficient understanding of the actual process and conditions in daily practice and how health care professionals adjust proceedings according to variable working conditions. FRAM is a method to visualize essential activities of the work-as-done including the variability of daily practice (Hollnagel et al., 2014).

To the best of our knowledge, a FRAM analysis of the double check proceeding during injectable medication administration has not yet been published by others. Therefore, we used FRAM to determine the fit between the double checking proceeding according to the protocol (work-as- 
Schutijser, B.C.F.M., Jongerden, I.P., Klopotowska, J.E., Portegijs, S., Bruijne, M.C. de, Wagner, C.Double checking injectable medication administration: Does the protocol fit clinical practice? Safety Science: 118 (2019) p. 853-860

imagined) and clinical practice by nurses (work-as-done) in order to learn about variation in the process, adapting behaviors of nurses and identify facilitators and barriers to correct performing the double check. This knowledge is needed in order to optimize safety during the injectable medication administration process.

\section{Materials and methods}

\subsection{Design}

Between February and July 2018, we conducted a qualitative study, using observations and semistructured (group) interviews focusing on the current daily practice of the double check during injectable medication administration. We defined the double check according to the Dutch protocol: a second nurse checks the administration on right (1) medication order, (2) medication name, (3) dose, (4) administration route, (5) administration rate, (6) patient and (7) time (VMS, 2009). The second nurse needs to compare the information with the original medication order from the physician (confirmation), and calculate the right administration rate separately (independent check). Hence, the second nurse has not only a confirmative role (Furniss et al., 2018). The medical ethical committee of the Amsterdam UMC, Vrije Universiteit Amsterdam waived the requirement of informed consent (protocol number: 2018/156) as they found the scope of the study outside the Dutch Medical Research (Human Subjects) Act. Nevertheless, prior to each observation and (group) interview, oral consent was obtained from the nurses. The COREQ guideline was used to report this study (Tong et al., 2007).

\subsection{Setting}

Two Dutch hospitals participated in this study, one university hospital (hospital A) and one general hospital (hospital B). The university hospital has 733 beds and over 7000 staff members and the general hospital has 277 beds and over 1500 staff members. Both hospitals were selected based on their compliance with the proceeding "double check by a second nurse", as evaluated in a previous evaluation study (Schutijser et al., 2018). Hospitals were compliant with the double check when all 7 steps (medication order, medication name, dose, administration route, administration rate, patient and time) were conducted correctly. Thus, when work-as-done was conducted according to work-asimagined. Since we also aimed to learn from what goes right, we selected one hospital with high compliance ( $100 \%$, hospital B) and one hospital with average compliance $(41 \%$, hospital A). In both hospitals, two wards were invited to participate, an internal medicine and (general) surgery ward. All four wards participated in the previous evaluation study (Schutijser et al., 2018).

\subsection{Participants}

For observations and interviews, all nurses (including trainees) involved in the administration of injectable medication on the participating wards were eligible to participate. Nurses were purposively invited to participate in the study by a senior nurse. For both the observations and interviews, nurses needed to be working on one of the selected wards and needed to be qualified to administer injectable medication. Participation in the study was voluntary for nurses and their information was anonymously processed.

\subsection{Functional resonance analysis method}

The Functional Resonance Analysis Method (FRAM) was used to visualize essential activities of the double check process as it usually takes place (Hollnagel et al., 2014). This "work-as-done" model can be compared with the ideal model according to the protocol ("work-asimagined"). The FRAM also takes into account variability, dependencies of activities and potential facilitators and barriers to correct performing the double check (Clay-Williams et al., 2015). For each essential activity, a 
Schutijser, B.C.F.M., Jongerden, I.P., Klopotowska, J.E., Portegijs, S., Bruijne, M.C. de, Wagner, C.Double checking injectable medication administration: Does the protocol fit clinical practice? Safety Science: 118 (2019) p. 853-860

function (visualized as a hexagon) is created. Functions are described by a verb and need to be performed by nurses to achieve a certain goal (i.e. to identify the patient). On each function, six aspects (Input, Output, Precondition, Resource, Control, Time) are relying (Fig. 1) (Hollnagel et al., 2014):

- Input: start of the function, for example the medication order is registered.

- Output: the result of the function, for example the administration of medication.

- Precondition: without these, the function cannot be conducted, for example the medication order is authorized.

- Resource: these are necessary during the execution of the function, for example working Wi-Fi connection to use a computer-on-wheels (COW) with a barcode scanner.

- Time: any time related restriction, for example, medication is only administered during the medication rounds.

- Control: the way the function is monitored, for example an audit every two months.

\section{[Figure 1]}

We used FRAM because the role of nurses can be seen as flexible and compensating. Nurses are often compensating for inadequacies in health care. This resilience results in adjusting to situations in practice (McNamara and Fealy, 2010). The number of studies that used FRAM in healthcare processes are limited (Buikstra, 2018; Clay-Williams et al., 2015; Damen et al., 2018; Laugaland et al., 2014; McNab et al., 2018; Raben et al., 2018; Ross et al., 2018). FRAM provides a unique opportunity to visualize complex processes. As compared to previous studies, FRAM can reveal which activities nurses actually do when they are double checking injectable medication. By visualizing this process with FRAM and the variability in the process, we aimed to better understand factors affecting the double check process. Besides, although individual interviews are recommended for the datacollection in FRAM studies, few studies conducted group interviews as a new method (Bridges et al., 2018; McNab et al., 2018). In our study, the work-as-done model was also constructed based on group interviews. The work-as-imagined model was based on the prevailing Dutch protocol for safe injectable medication administration (VMS, 2009).

Functions in both work-as-imagined and work-as-done models were further divided in "foreground" and "background" functions: foreground functions were defined as the main and most important activities related to the double checking process. Background functions are activities related to other proceedings during injectable medication administration. Facilitators and barriers to correct performing the double check were divided in culture, technology, staff and organizational related factors and were based on a data-driven analysis. In addition, to better understand the workas-done models, variability of the Output of foreground functions were described in (1) internal variability (i.e. variability of the function itself), (2) external variability (i.e. variability of the work environment), (3) upstream-downstream coupling (i.e. variability from upstream functions), and (4) the manifestations of variability in terms of time (too early/on time/too late/omission) and precision (imprecise/acceptable/precise) (Hollnagel et al., 2014). Functions with a high variability can have impact on other functions further in the FRAM model and eventually involve additional risks for conducting the process.

\subsection{Data collection}

We first conducted observations of nurses during injectable medication administration followed by semi-structured (group) interviews with nurses. Nurses were directly observed by using the same method as in previous observational studies by our research group (Schilp et al., 2014; Schutijser et al., 2018). In summary, nurses were observed while administering injectable medication to patients 
Schutijser, B.C.F.M., Jongerden, I.P., Klopotowska, J.E., Portegijs, S., Bruijne, M.C. de, Wagner, C.Double checking injectable medication administration: Does the protocol fit clinical practice? Safety Science: 118 (2019) p. 853-860

older than 18 years, during the medication rounds from 6 AM to 10 PM. Parenteral nutrition, intravenous chemotherapy and acute medications were excluded from the observations because for these medications other administration protocols apply. A standardized observation form was used to evaluate the nine most important and identifiable administration proceedings from the prevailing Dutch protocol (Supplement A). Correctly conducted proceedings were marked on the observation form. The findings from the observations served only as input for the interviews to give examples of proceedings with high and low compliance. Observations were not further analyzed, as no differences in the observations were found in comparison to the previous observational study (Schutijser et al., 2018).

After the observations, nurses were interviewed either individually or in groups of two or three nurses. The group size depended on the availability of the nurses. For the interviews, a topic list was used, which was based on the FRAM method (Supplement B). In order to gain rich and meaningful data, examples from observations were used during the interviews and nurses were invited to reflect on these observations. One researcher (BS) conducted the interviews and a second researcher (SP) assisted and made field notes. Each (group) interview lasted approximately $30 \mathrm{~min}$ and started with an introduction of the researchers and the goal of the interview. Also, gender and nursing experience (in years) of the participants were registered. Interviews were recorded if participants gave their consent. Two nurses refused to be recorded, one due to medical and one due to personal reasons; those interviews were transcribed directly after the interview by both researchers. Interviews were executed until data saturation was reached, providing no new information regarding the wards process of double checking. Two test interviews with independent nurses were conducted to gain more experience with the interview questions and to finalize the topic list.

\subsection{Data analysis}

All interviews were transcribed within a few days following the interview. Both researchers (BS and SP) individually coded all interviews through open coding and by using the six FRAM aspects as codes. Every process step during the double check was translated to a function. Then, related aspects (Input, Output, Precondition, Resource, Control, Time) were recognized from and coded per ward. Two senior researchers (IJ and JK) also coded the first two interviews in order to reach consensus about the coding method. After coding the interviews, the work-as-done model was first drafted in a FRAM model on paper, then discussed by BS, SP and IJ, and finally created with the FRAM Model Visualizer software (Hill, Version 0.4.1 2016). Two overall work-as-done models were created representing the aggregated processes in both hospitals. After the data analysis, each ward was offered the opportunity to discuss the results (in a meeting) from the work-as-done model and to formulate recommendations to help eliminate identified barriers.

\section{Results}

\subsection{Participants}

In total, 18 nurses were observed during injectable medication administration: 10 nurses in hospital $A$ and 8 nurses in hospital B. Subsequently, 27 nurses were interviewed during 15 interviews (Table 1). Most interviewed nurses were female $(81 \%)$ and median years of nursing experience was 8 years (Inter Quartile Range: 4-13 years). In both participating hospitals, nurses used electronic health records (EHR) with medication administration records and barcode medication administration (BCMA) systems during the injectable medication administration process. Data saturation was reached on all wards. 
Schutijser, B.C.F.M., Jongerden, I.P., Klopotowska, J.E., Portegijs, S., Bruijne, M.C. de, Wagner, C.Double checking injectable medication administration: Does the protocol fit clinical practice? Safety Science: 118 (2019) p. 853-860

\section{[Table 1]}

\section{[Figure 2]}

\subsection{Work-as-imagined model using FRAM}

The work-as-imagined model consisted of 23 functions of which 2 foreground functions focused specifically on the double check (Fig. 2):

1. To administrate medication: The medication is prepared and ready for administration (Input). The medication needs to be administered at the prescribed Time. The nurse asks a second nurse for a physical double check at the patients' bedside (from Output of $<$ To administrate medication $>$ to Input of $<$ To conduct the double check $>$ ). The Output is that the medication is administered and can be signed off (from Output of $<$ To administrate medication $>$ to Input of $<$ To sign medication order $>$ ).

2. To conduct the double check: The second nurse checks the medication order, medication name, dose, administration route, administration rate, patient and time. The double check is considered 'complete' when all 7 steps are conducted (Supplement A) (from Output of $<$ To conduct the double check $>$ to Control of $<$ To administrate medication $>$ ). A Precondition for the double check is that the medication has been ordered by a physician.

\subsection{Work-as-done model for hospital A using FRAM}

In hospital A, we identified 3 additional foreground functions as compared to the work-as-imagined model. Besides, 1 of the 2 foreground functions from the work-as-imagined model was adapted in daily practice. In total, 5 foreground functions focused specifically on the double check (Fig. 3):

\section{[Figure 3]}

1. To assess the situation on the ward: Medication is prepared and ready for administration (Input). Nurses assess the situation on the ward by mentally considering several criteria (Control): how frequent he/she administrated this medication previously, what the current workload is on the department, how severe the possible side effects of the medication can be, what the current medical condition of the patient is and what type of medication need to be administered by which administration route. The Output is a decision whether a second nurse is needed to conduct a physical double check.

(IA3): "My instinct tells me that medication administered by syringe pumps, potassium and antibiotics are really high-risk medications. Therefore, I find it necessary to involve a second nurse in the double check."

(IA6): "Anti Thymocyte Globulin for example, that is a medication with many side effects. For me, that is a high-risk medication and I handle it extra carefully. Not only during preparation, but also during administration a double check from a colleague is necessary."

2. To verify administration route and rate in the hallway or medication room: If the nurse decides that the physical double check is unnecessary, but a second nurse happens to be available in the medication room or in the hallway, the right administration route and rate will be verified orally. If no colleague nurse is available in the medication room or hallway and/or when the nurses decide that no physical double check is necessary, then the nurse proceeds to the patients' bedside.

(IA8): "I take the medication with me and then I show it to another nurse who usually stands in the hallway with the Computer on Wheels (COW). 'I'm now going to give this medication to that patient, is that ok?' and then I sign for the medication and go to the patient." 
Schutijser, B.C.F.M., Jongerden, I.P., Klopotowska, J.E., Portegijs, S., Bruijne, M.C. de, Wagner, C.Double checking injectable medication administration: Does the protocol fit clinical practice? Safety Science: 118 (2019) p. 853-860

3. To conduct digital double check: The nurse walks to the patients' bedside with a COW and a barcode scanner (Resources). Through the use of a COW, the nurse has access to the medication administration record within the EHR of the patient. The nurse executes a digital double check for 5 of the 7 steps: right patient by scanning the barcode on the wristband of the patient and right medication order, name, dose and time by scanning the barcode on the medication label. The information from the wristband and medication label is automatically compared with the information in the HER and medication administration record. If discrepancies are identified, the nurse is alerted by a pop-up.

4. To administrate medication: When the digital double check has been conducted and no discrepancies have been identified, the nurse starts to administer the medication (Input). A Precondition is that the infusion is adequately running and during the administration all materials are available (Resource). When the nurse decides a physical double check is necessary, a second nurse is asked to accompany him or her. The Output is that the medication is administered.

5. To conduct physical double check: A second nurse conducts only the final 2 steps of the double checking proceeding: right administration route and rate. During the interviews, nurses provided examples of medications for which they think a physical double check is always necessary: heparin, morphine, midazolam, ketamine, potassium, furosemide, insulin and infliximab. In addition, nurses mentioned that the physical check is more feasible during dayshifts than during evening or nightshifts (Time) since during dayshifts, enough staff is available to conduct the physical double check. Finally, it is possible for ward supervisors to digitally check if the physical double check has been signed in the system (Control).

\subsection{Work-as-done model for hospital B using FRAM}

The work-as-done in hospital B differed from hospital A only on the check of the right patient (Fig. 4). This is an extra foreground function in the model as composed to hospital A. Nurses at hospital B conduct almost always, regardless using a barcode scanner, a verbal verification of the patients' identity with the patient.

3. To identify the patient: In addition to the digital check on right patient, patients that are conscious (Precondition) are verbally asked for their date of birth. If a patient is unconscious, then the nurse starts the actual administration and this activity is skipped.

(IB5): At the patients' bedside you still check the patients' date of birth. You always ask that, you just have to."

In addition, during the interviews, nurses at hospital B provided examples of medications for which they think a physical double check is always necessary: prothrombin complex concentrate, gentamicin, heparin, morphine, midazolam and blood.

\subsection{Differences between hospital A and B}

In addition to the identification of the patient in the work-as-done model in hospital $B$, there are several other differences between both hospitals. In contrast to hospital $A$, the pharmacy department in hospital B prepares almost all scheduled injectable medication (mainly antibiotics) and nurse trainees are allowed to conduct both the digital and physical double check (after they have passed a test). Furthermore, the presence of a second nurse in hospital $B$ is experienced as an interruption by some nurses, making it less likely that they ask a second nurse for the physical double check. On the other hand, if nurses in hospital A decided that no second nurse is needed/available for the physical double check, they still verify the administration route and rate with another nurse on the wards' hallway. 
Schutijser, B.C.F.M., Jongerden, I.P., Klopotowska, J.E., Portegijs, S., Bruijne, M.C. de, Wagner, C.Double checking injectable medication administration: Does the protocol fit clinical practice? Safety Science: 118 (2019) p. 853-860

(IB1): "With a nurse trainee you are already with two and when the trainee administers the medication, I can check him/her."

\subsection{Variability}

Variability was mainly identifiable in the function: $<$ To assess the situation on the ward $>$ as this assessment is conduced depending on the opinion of a nurse involved in injectable medication administration. The criteria used for this assessment resulted in the decision whether or not a second nurse is asked for the physical double check. This decision is individually made (internal variability) and seems to relate to the subjective expertise, experience and judgement of the nurse involved. The decision either results in the execution of the physical double check further in the process or not $(<$ To conduct physical double check $>$ ). External variability for this function seems to relate to staffing (interns available or not), the type of department, the complexity of patients diseases on the department and to unspoken expectations between nurses regarding the assessment. The imprecise output of this function can affect the variability of downstream functions (upstream-downstream coupling). To understand the manifestations of variability in terms of time and precision; the assessment is conducted during the administration and is therefore on time. However, the outcome of the decision is imprecise due to each nurses' interpretation of the situation at that moment.

\section{[Figure 4]}

\subsection{Facilitators and barriers}

Facilitators and barriers to correct performing the double check can be divided into culture, technology, staff and organizational related factors. Most nurses understood why the double check is necessary and that it contributes to decreasing the amount of medication errors. Moreover, nurses believe that their team is critical, collegial and open for feedback. Electronic health records with medication administration records and barcode systems support the double check. Additionally, the availability of trainees increases the opportunity for the physical double check, since it provides more personnel. A final facilitator is that the hospital pharmacy prepares several types of medication.

However, there are also barriers. A small proportion of the nurses did not believe that the double check contributes to improved medication safety. Besides, nurses experience an increased workload due to increased clinical complexity of patient care and short admission periods. Furthermore, the COW sometimes fails to work resulting in delays in the medication administration process. Mainly, a staff shortage, in addition to a lack of time and the amount of interruptions during injectable medication administration was mentioned. A final barrier was lacking an overview of the medication preparation process by the hospital pharmacy.

(IB2): "Officially, two nurses have to go to the patient, but that does not always happen, especially due to a lack of time."

(IB5): "I have never succeeded in giving medication in a quiet environment."

(IB3): "In the past, maybe 5 patients on the ward had an infusion, now almost everyone has one."

\section{Discussion}

In our study, we found a lack of fit between work-as-done and workas- imagined in double checking during injectable medication administration. The work-as-done model is more complex and consists of more activities than the work-as-imagined model. During work-as-done, nurses split the double check into a digital and physical check. The digital check is a double check on right medication order, medication, dose, time and patient. The physical check is a double check on right administration route and rate. Nurses almost always succeed in conducting the digital double check, 
Schutijser, B.C.F.M., Jongerden, I.P., Klopotowska, J.E., Portegijs, S., Bruijne, M.C. de, Wagner, C.Double checking injectable medication administration: Does the protocol fit clinical practice? Safety Science: 118 (2019) p. 853-860

but not always in conducting the physical double check for every administration. Although the intention to conduct the physical double check is certainly present among nurses, several barriers result in protocol workarounds. For example, prior to the administration, nurses conduct their own risk-assessment (using various criteria) to decide whether or not a physical double check is absolutely necessary in their opinion.

This study visualized the double check process of nurses in detail. Only few previous studies focused in such detail on the double check as well, which makes our results innovative and complementary to existing results. Most studies reported that the double check process is poorly defined and that many variations of the double check process are executed in hospitals (Dickinson et al., 2010; Kellett and Gottwald, 2015). One detailed process description is the read-read back method from (Schwappach et al., 2016): one nurse reads out loud the medication order and another nurse verifies this on the medication label and vice versa. However, this study is different from ours since it is conducted on an oncology ward and focused on chemotherapy, and the role of the barcode scanner is unclear in the various scenario's.

We found that a lack of time is a major barrier for conducting the double check proceeding during injectable medication administration. This is in line with other studies (Alsulami et al., 2012; Armitage, 2008; Schwappach et al., 2018). The double check, in particular the physical double check, is time-consuming and requires a second nurse at the patients' bedside (Schutijser et al., 2018). With the implementation of BCMA systems, time spent on double checking has already been reduced. In hospitals with a BCMA system, the double check can always be conducted digitally for 5 of the 7 double check steps. Therefore, the risk of patient harm is already reduced during the double check process. Should it be possible to conduct the other 2 steps, right administration route and rate, by also using the barcode scanner (for example with smart pumps or a camera in the barcode scanner), then the time spent on the double check will be reduced dramatically and the risk of patient harm will reduce further. Other facilitators to correct performing the double check, according to the nurses, that reduce the time spent on double checking are the availability of trainees and the preparation of injectable medication by the hospital pharmacy. Shifting the preparation of injectable medication from nurses on the ward to the hospital pharmacy is an emerging development. It is even estimated that using robotic devices to prepare medications in the hospital pharmacy can reduce healthcare costs and medication errors (Urbine and Schneider, 2014).

Nurses assess the situation on the ward because of a lack of time and availability of a second nurse to conduct the physical double check for all injectable medication administrations. Criteria used for this assessment are not mentioned in previous studies, except the patients' medical condition and the type of medication (Alsulami et al., 2012; Kellett and Gottwald, 2015). The individual assessment by nurses can be seen as a positive and negative outcome of the lack of fit between the workas- imagined and work-as-done. Positive because it shows the nurses' willingness to decrease risk of patient harm when administering injectable medication by using their knowledge about and experience with medication. Based on this knowledge and experience, nurses make a wellconsidered and substantiated decision to work around the protocol. The physical double check of 'top high-risk' medication can be seen as a good practice from which we can learn. Not following the protocol completely without making a medication error might reduce work load and fit better in practice. However, the down side of the assessment is that it relies on individual experience, knowledge and confidence of the nurse involved. Therefore, it introduces a high degree of variability in the double check process. The current protocol does not accept variability and requires that all 7 steps included in the double check proceeding are conducted independently by a second nurse for all injectable medications. Thus, if the protocol is leading, the assessment is not desirable. However, at present it is unknown if the individual assessment by nurses actually leads to errors in the injectable medication administration process. Future research should focus on this aspect and reconsider whether to continue to pursue $100 \%$ protocol compliance or accepting that variation exist and that 
Schutijser, B.C.F.M., Jongerden, I.P., Klopotowska, J.E., Portegijs, S., Bruijne, M.C. de, Wagner, C.Double checking injectable medication administration: Does the protocol fit clinical practice? Safety Science: 118 (2019) p. 853-860

nurses assess the situation on the ward. Besides, future research can also focus on whether the double check need to be independent or that a confirmative role is also sufficient.

To date, only a limited number of studies applied FRAM to evaluate healthcare processes (Buikstra, 2018; Clay-Williams et al., 2015; Damen et al., 2018; Laugaland et al., 2014; McNab et al., 2018; Raben et al., 2018; Ross et al., 2018). We believe FRAM is very useful for visualizing the complexity of healthcare processes, such as the double check. The (group) interviews appeared an effective method to gain detailed information about the work-as-done. In the future, studies focusing on this topic may learn from methodological enhancements from other FRAM studies, for example by conducting the FRAM in more than one country (Damen et al., 2018), by analyzing the process backwards (Bridges et al., 2018), or by creating an intervention based on the FRAM models (Ross et al., 2018). Furthermore, Shorrocks' extra dimensions work-as-disclosed and work-asprescribed describe a grey area that can be explored more while balancing between visualizing the complex process and the ease of interpretation for nurses (Shorrock, 2016). In total, the time investment in our study to conduct the FRAM per hospital was approximately $60 \mathrm{~h}$. This is more than the suggested time investment of Damen et al. (2018), because we also conducted observations at each site (Damen et al., 2018). The two selected hospitals differed in hospital type (general vs. university) and double check compliance rate (high vs. average compliance). A striking finding in our study is that, despite the major differences in compliance rates between the participating hospitals in the previous study (Schutijser et al., 2018), the differences in the actual work-as-done process in both hospitals were small. Three factors may explain why the observed compliance in hospital B was higher in the previous study (Schutijser et al., 2018). Firstly, the Hawthorne effect during observations may have had more influence on the performance of injectable medication administration than we initially thought. The interviews showed that nurses know all protocol proceedings for safe injectable medication administration considerably well and are able to reproduce them if needed. Secondly, in hospital B, trainees were allowed to conduct both the digital and physical double check which works effectively in overcoming a staff shortage. Thirdly, hospital A had a higher amount of injectable medication administrations per medication round. When many medications needs to be administered at the same time by several nurses, it is certainly plausible that they have less time to conduct the physical double check.

\subsection{Strengths and limitations}

To our knowledge, this is the first study that used FRAM to provide detailed information about the actual clinical process of double checking injectable medication. We determined the double check process by using a combination of observations and interviews. Since the double check is, preeminently, a proceeding that requires the cooperation of more than one nurse, we used the power of group interviews to invite nurses to respond to each other. However, this study also has some limitations. Firstly, this study was limited to two different wards in two hospitals. Therefore, the results cannot be generalized to all hospitals. Secondly, nurses in our sample were invited in a purposive sampling way. This may have brought bias in the results, as the nurses could be a selected group or could be the ones with the most outspoken opinions about the double check proceeding. However, none of the nurses asked, refused to participate. Moreover, the gender and years of nursing experience varied between the nurses, which suggest that our sample was a heterogeneous group of nurses.

\section{Conclusions}

By using FRAM, we identified a lack of fit between work-as-imagined and work-as done for conducting the double check proceeding during injectable medication administration in two Dutch hospitals. Work-as-done revealed that, prior to conducting the double check, nurses assess the patients' and wards' situation and decide whether a physical double check is necessary or not. A 
Schutijser, B.C.F.M., Jongerden, I.P., Klopotowska, J.E., Portegijs, S., Bruijne, M.C. de, Wagner, C.Double checking injectable medication administration: Does the protocol fit clinical practice? Safety Science: 118 (2019) p. 853-860

physical double check is most likely conducted when a patient is vulnerable or when the medication is not frequently administered. The risk-impact analysis is individually made and may vary between nurses. It is unknown whether this variability causes patient harm. It is important to reconsider to what extent practice variation is acceptable and safe, or that the focus will still be on $100 \%$ protocol compliance. If variation due to the assessment by nurses is to be accepted, then we recommend to organize discussion meetings among nurses and the ward management to raise awareness about the assessment, the criteria and barriers and risks. Furthermore, we recommend ensuring adequate education of nurses to achieve the individual assessment. Future research should focus on the possibilities for conducting the double check solely digitally by using a barcode scanner.

\section{Declaration of Competing Interest}

None.

\section{Acknowledgement}

We would like to thank all nurses who participated in this study and the staff of all wards for their cooperation. Furthermore, we want to thank Tanja Mol, MSc for performing a test interview and Marco Moesker, MSc for his advice and experience with the FRAM methodology and for performing a test interview. Finally, we want to thank Catherine Combee - Duffy, MANP for improving the readability of this article.

\section{Funding}

This study was funded by the Dutch Ministry of Health, Welfare, and Sports.

\section{Appendix A. Supplementary material}

Supplementary data to this article can be found online at https:// doi.org/10.1016/j.ssci.2019.06.026.

\section{References}

Alemanni, J., Touzin, K., Bussieres, J.F., Descoteaux, R., Lemay, M., 2010. An assessment of drug administration compliance in a university hospital centre. J. Eval. Clin. Pract. 16, 920-926.

Alsulami, Z., Choonara, I., Conroy, S., 2014. Paediatric nurses' adherence to the doublechecking process during medication administration in a children's hospital: an observational study. J. Adv. Nurs. 70, 1404-1413.

Alsulami, Z., Conroy, S., Choonara, I., 2012. Double checking the administration of medicines: what is the evidence? A systematic review. Arch. Dis. Child. 97, 833-837.

Armitage, G., 2008. Double checking medicines: defence against error or contributory factor? J. Eval. Clin. Pract. 14, 513-519.

Barthelemy, I., Huet, E., Guilbeault, M., Bussieres, J.F., 2013. Compliance with inpatient drug use process steps: a cross-sectional observational study. J. Pharm. Pract. 26, 551-555.

Bridges, K.E., Corballis, P.M., Hollnagel, E., 2018. “Failure-to-Identify” hunting incidents: a resilience engineering approach. Hum. Fact. 60, 141-159.

Buikstra, E., 2018. Using FRAM to explore Medication Administration Processes: FRAM as a Learning Tool. Available from: http://functionalresonance.com/onewebmedia/ 8\%20Buikstra\%20Presentation\%20FRAMily\%2013\%20June\%202018.pdf (accessed on 10 Oct. 2018).

Choo, J., Johnston, L., Manias, E., 2013. Nurses' medication administration practices at two Singaporean acute care hospitals. Nurs. Health Sci. 15, 101-108.

Clay-Williams, R., Hounsgaard, J., Hollnagel, E., 2015. Where the rubber meets the road: using FRAM to align work-as-imagined with work-as-done when implementing clinical guidelines. Implem. Sci. 10, 125.

Damen, N.L., de Vos, M.S., Moesker, M.J., Braithwaite, J., de Lind van Wijngaarden, R.A.F., Kaplan, J., Hamming, J.F., Clay-Williams, R., 2018. Preoperative anticoagulation management in everyday clinical practice: an international comparative analysis of work-as-done using the Functional Resonance Analysis Method. J. Pat. Saf. 
Schutijser, B.C.F.M., Jongerden, I.P., Klopotowska, J.E., Portegijs, S., Bruijne, M.C. de, Wagner, C.Double checking injectable medication administration: Does the protocol fit clinical practice? Safety Science: 118 (2019) p. 853-860

Dickinson, A., McCall, E., Twomey, B., James, N., 2010. Paediatric nurses' understanding of the process and procedure of double-checking medications. J. Clin. Nurs. 19, 728-735.

Furniss, D., Lyons, I., Franklin, B.D., Mayer, A., Chumbley, G., Wei, L., Cox, A.L., Vos, J., Galal-Edeen, G., Blandford, A., 2018. Procedural and documentation variations in intravenous infusion administration: a mixed methods study of policy and practice across 16 hospital trusts in England. BMC Health Serv. Res. 18, 270.

Hill, R., 2016. FRAM Model Visualizer. Available from: http://functionalresonance.com/ FMV/index.html (accessed on 1 Jul. 2018).

Hollnagel, E., Hounsgaard, J., Colligan, L., 2014. FRAM - the Functional Resonance Analysis Method - a handbook for the practical use of the method. Available from: http://functionalresonance.com/onewebmedia/FRAM_handbook_web-2.pdf (accessed on 14 Aug. 2018).

Hughes, R.G., Blegen, M.A., 2008. Medication administration safety. In: Hughes, R.G. (Ed.), Patient safety and quality: an evidence-based handbook for nurses. Agency for Healthcare Research and Quality, Rockville MD.

Institute for Safe Medication Practices (ISMP), 2015. Safe practice guidelines for adult IV push medications. A compilation of safe practices from the ISMP adult IV push medication safety summit. Available from: http://www.ismp.org/Tools/guidelines/ ivsummitpush/ivpushmedguidelines.pdf (accessed on 9 Aug. 2018).

Kellett, P., Gottwald, M., 2015. Double-checking high-risk medications in acute settings: a safer process. Nurs. Manage. (Harrow) 21, 16-22.

Laugaland, K., Aase, K., Waring, J., 2014. Hospital discharge of the elderly-an observational case study of functions, variability and performance-shaping factors. BMC Health Serv. Res. 14, 365.

McDowell, S.E., Mt-Isa, S., Ashby, D., Ferner, R.E., 2010. Republished paper: where errors occur in the preparation and administration of intravenous medicines: a systematic review and Bayesian analysis. Postgrad. Med. J. 86, 734-738.

McNab, D., Freestone, J., Black, C., Carson-Stevens, A., Bowie, P., 2018. Participatory design of an improvement intervention for the primary care management of possible sepsis using the Functional Resonance Analysis Method. BMC Med. 16, 174.

McNamara, M.S., Fealy, G.M., 2010. Guest editorial: lead us not again: clinical leadership and the disciplinary contribution. J. Clin. Nurs. 19, 3257-3259.

Raben, D.C., Bogh, S.B., Viskum, B., Mikkelsen, K.L., Hollnagel, E., 2018. Learn from what goes right: a demonstration of a new systematic method for identification of leading indicators in healthcare. Reliab. Eng. Syst. Saf. 169, 187-198.

Ross, A., Sherriff, A., Kidd, J., Gnich, W., Anderson, J., Deas, L., Macpherson, L., 2018. A systems approach using the functional resonance analysis method to support fluoride varnish application for children attending general dental practice. Appl. Ergon. 68,294-303.

Schilp, J., Boot, S., de Blok, C., Spreeuwenberg, P., Wagner, C., 2014. Protocol compliance of administering parenteral medication in Dutch hospitals: an evaluation and cost estimation of the implementation. BMJ Open 4, e005232.

Schutijser, B., Klopotowska, J.E., Jongerden, I., Spreeuwenberg, P., Wagner, C., de Bruijne, M., 2018. Nurse compliance with a protocol for safe injectable medication administration: comparison of two multicentre observational studies. BMJ Open 8, e019648.

Schwappach, D.L., Pfeiffer, Y., Taxis, K., 2016. Medication double-checking procedures in clinical practice: a cross-sectional survey of oncology nurses' experiences. BMJ Open 6, e011394.

Schwappach, D.L.B., Taxis, K., Pfeiffer, Y., 2018. Oncology nurses' beliefs and attitudes towards the doublecheck of chemotherapy medications: a cross-sectional survey study. BMC Health Serv. Res. 18, 123.

Sheu, S.J., Wei, I.L., Chen, C.H., Yu, S., Tang, F.I., 2009. Using snowball sampling method with nurses to understand medication administration errors. J. Clin. Nurs. 18, 559-569.

Shorrock, S., 2016. The Varieties of Human Work. Available from: https:// humanisticsystems.com/2016/12/05/the-varieties-of-human-work/ (accessed on May 15, 2019).

Tong, A., Sainsbury, P., Craig, J., 2007. Consolidated criteria for reporting qualitative research (COREQ): a 32item checklist for interviews and focus groups. Int. J. Qual. Health Care 19, 349-357.

Urbine, T.F., Schneider, P.J., 2014. Estimated cost savings from reducing errors in the preparation of sterile doses of medications. Hosp. Pharm. 49, 731-739. 
Schutijser, B.C.F.M., Jongerden, I.P., Klopotowska, J.E., Portegijs, S., Bruijne, M.C. de, Wagner, C.Double checking injectable medication administration: Does the protocol fit clinical practice? Safety Science: 118 (2019) p. 853-860

VMS, 2009. High Risk Medication: Preparation and Administration of Parenteralia. Available from: https://www.vmszorg.nl/wp-content/uploads/2017/03/2009.0108 praktijkgids_high_risk.pdf (accessed on 9 Aug. 2018).

Westbrook, J.I., Rob, M.I., Woods, A., Parry, D., 2011. Errors in the administration of intravenous medications in hospital and the role of correct procedures and nurse experience. BMJ Qual. Saf. 20, 1027-1034.

\section{Tables and figures}

Figure 1 Example of a FRAM Function.

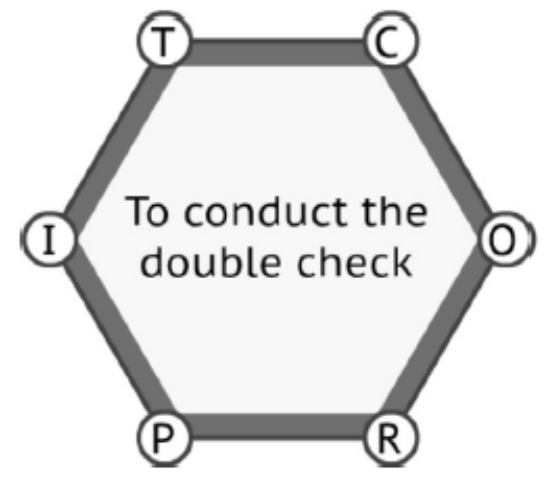

Table 1 Characteristics of the interviewed nurses $(n=27)$.

\begin{tabular}{|c|c|c|c|}
\hline & $\begin{array}{l}\text { Hospital A } \\
(n=13)\end{array}$ & $\begin{array}{l}\text { Hospital B } \\
(\mathrm{n}=14)\end{array}$ & Total $(n=27)$ \\
\hline \multicolumn{4}{|l|}{ Type of ward } \\
\hline $\begin{array}{l}\text { Internal Medicine, } \mathrm{n} \\
(\%)\end{array}$ & $7(54)$ & $8(57)$ & $15(56)$ \\
\hline $\begin{array}{l}\text { (General) Surgery, n } \\
(\%)\end{array}$ & $6(46)$ & $6(43)$ & $12(44)$ \\
\hline \multicolumn{4}{|l|}{ Gender } \\
\hline Male, n (\%) & $3(23)$ & $2(14)$ & 5 (19) \\
\hline Female, n (\%) & $10(77)$ & $12(86)$ & $22(81)$ \\
\hline \multicolumn{4}{|c|}{ Years of nursing experience } \\
\hline Median (IQR) & $8(5.5-9)$ & $7.5(4-36)$ & $8(4-13)$ \\
\hline
\end{tabular}


Schutijser, B.C.F.M., Jongerden, I.P., Klopotowska, J.E., Portegijs, S., Bruijne, M.C. de, Wagner, C.Double checking injectable medication administration: Does the protocol fit clinical practice? Safety Science: 118 (2019) p. 853-860

Figure 2 'Work-as-imagined' model for the injectable medication administration process.

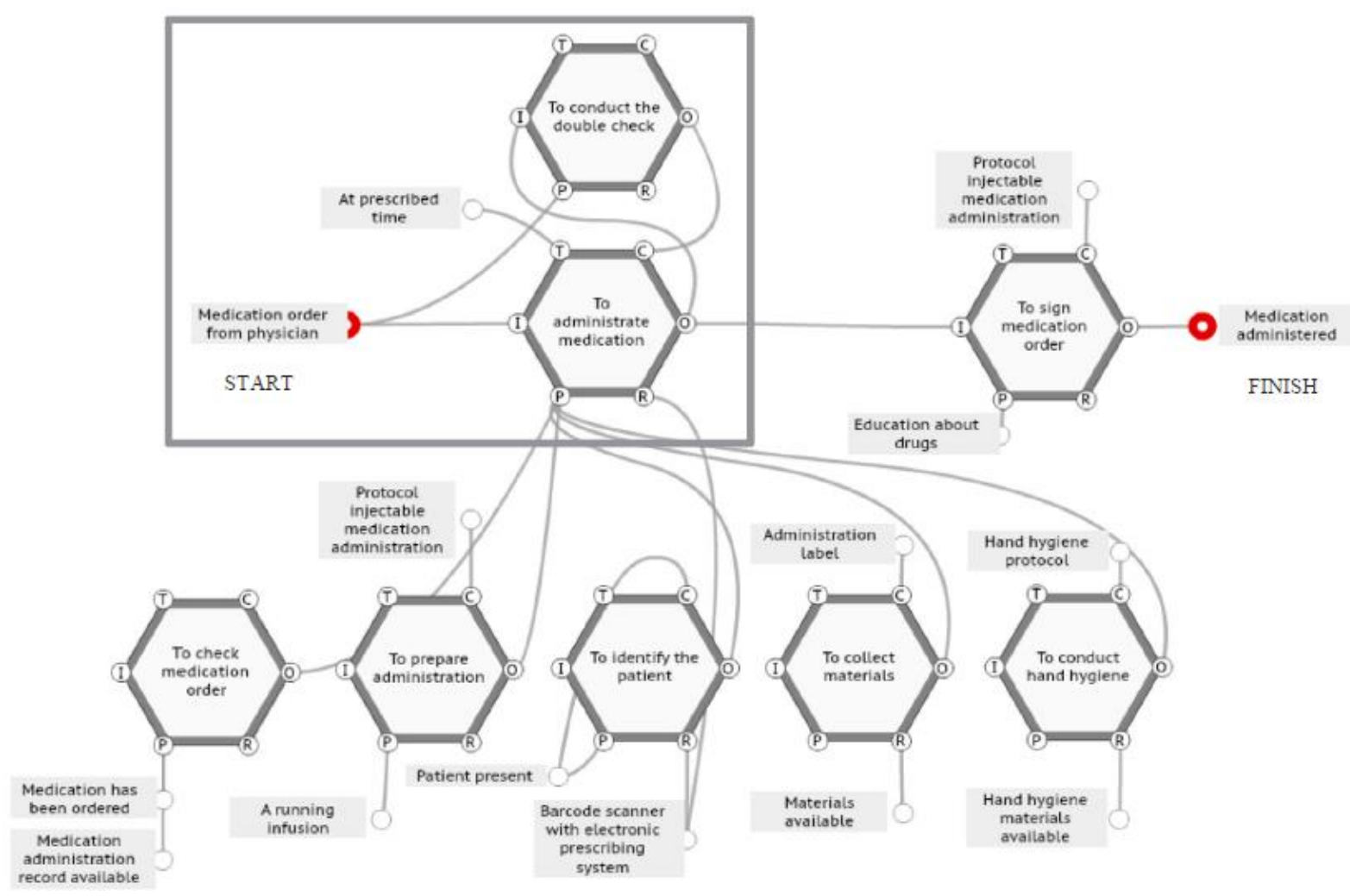

Figure 3 'Work-as-done' model for the double check procedure during injectable medication administration in hospital A. Black hexagons are new activities as compared to the 'workas-imagined' model.

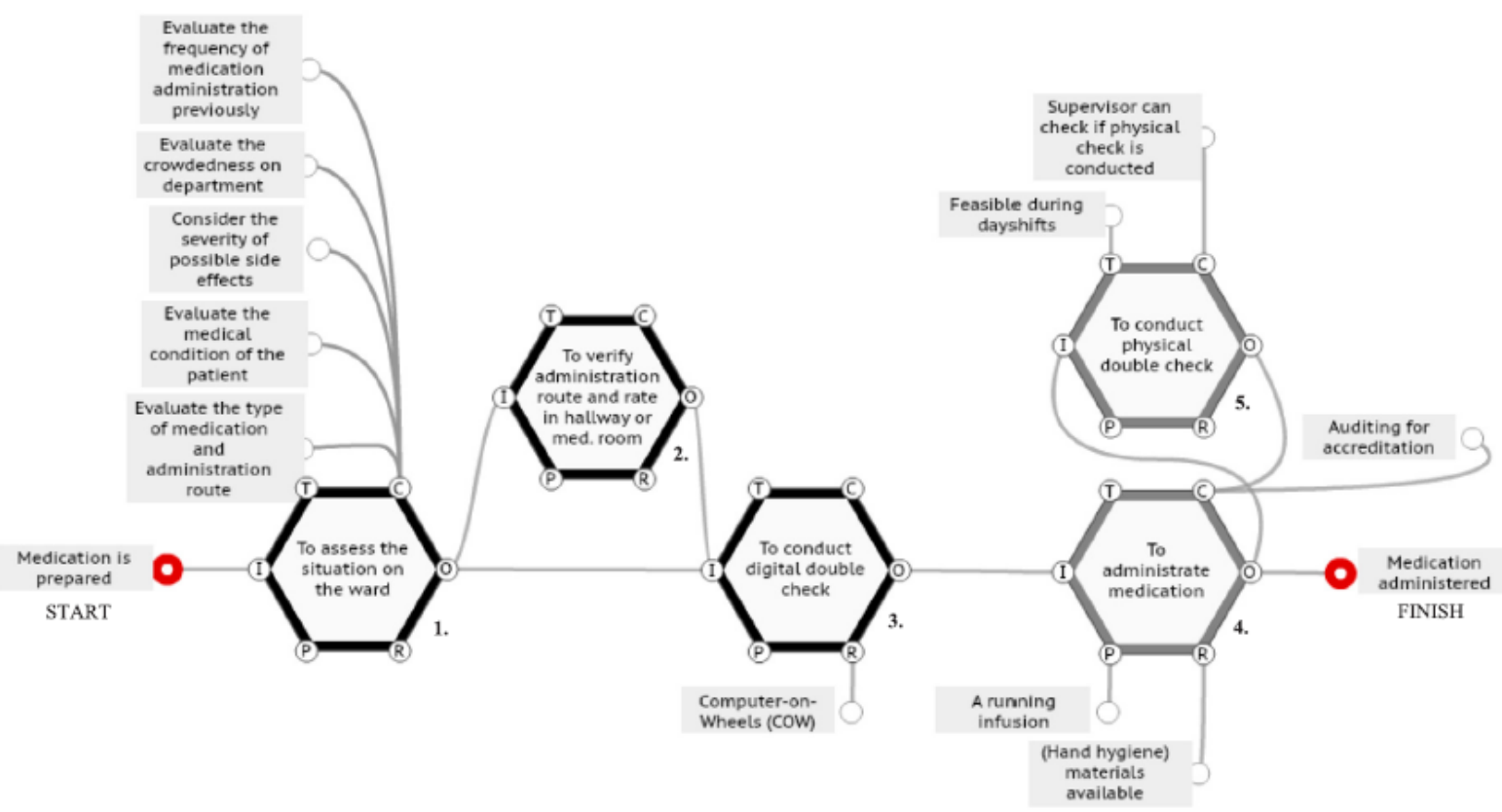


Schutijser, B.C.F.M., Jongerden, I.P., Klopotowska, J.E., Portegijs, S., Bruijne, M.C. de, Wagner, C.Double checking injectable medication administration: Does the protocol fit clinical practice? Safety Science: 118 (2019) p. 853-860

Figure 4. 'Work-as-done' model for the double check procedure during injectable medication administration in hospital B. Black hexagons are new activities as compared to the 'workas-imagined' model.

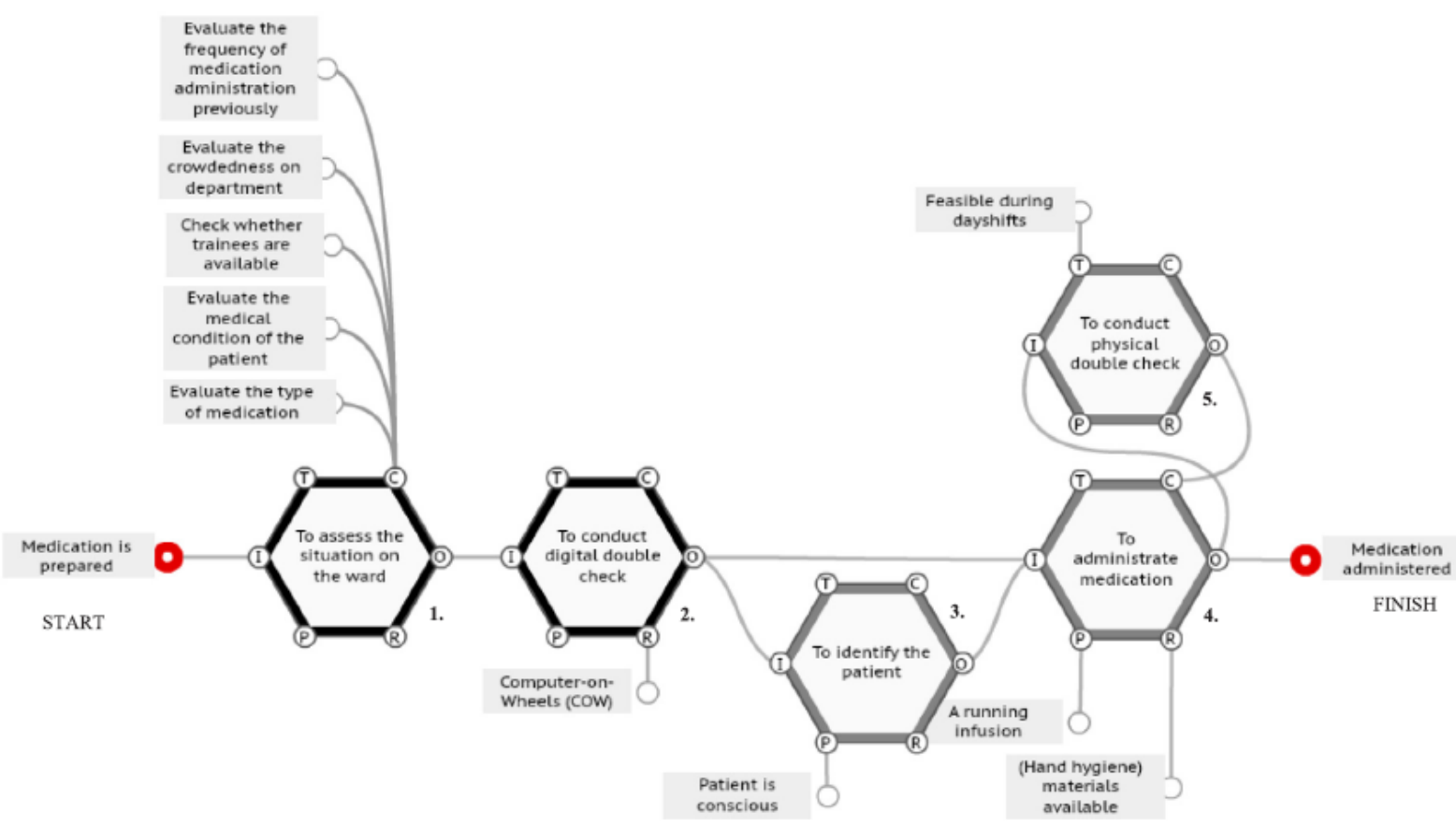

